\title{
Low-Frequency Radio Propagation into a Moderately Rough Sea
}

\author{
Donald F. Winter ${ }^{2}$
}

\section{Contribution from Applied Research Laboratory, Sylvania Electronic Systems, a Division of Sylvania Electric Products, Inc., 40 Sylvan Road, Waltham 54, Mass.}

(Received March 18, 1963)

\begin{abstract}
Radiation from primary current sources above or at the interface of a homogeneous conducting dielectric half space has been studied extensively in connection with various communications problems. In many cases of practical interest, however, the surface bounding the conducting medium is irregular in configuration, the local deviation of the interface from the average being a function of position and time. The present paper describes a method for determining the effect of interface irregularities upon low-frequency electromagnetic fields propagated within such a conductor.

The electric field at a point within the medium is expressed as a stochastic integral over the bounding surface using the method of Stratton and Chu. As a consequence of certain restrictions which are principally geometric in character, a perturbation technique can be employed to simplify the integrand. Following the introduction of assumptions regarding the statistical properties of the local surface deviations, approximate expressions are developed for the expectation of the subsurface field and the mean square deviation of the field amplitude. Numerical results for this latter quantity are obtained for the case of a vertical electric dipole as the radiating source on the interface between free space and a moderately rough sea. It is shown that sea surface irregularities can produce a pronounced effect upon subsurface fields in some instances; a tentative physical interpretation of the numerical results is advanced.
\end{abstract}

\section{Introduction}

Propagation of electromagnetic waves above and within a homogeneous conducting dielectric half space due to certain prescribed elementary (time harmonic) current sources has been studied by a number of workers beginning with Sommerfeld [1909]. Modifications of Sommerfeld's original work by Weyl [1919] and Sommerfeld [1926], together with more recent extensions by Norton [1937], Baños and Wesley [1954], Wait [1959a], Moore and Blair [1961], and many others, comprise a fairly exhaustive treatment of electric and magnetic dipole radiation in the presence of a semi-infinite conducting medium.

In many situations of practical interest, the surface separating free space from the conducting medium is not smooth. In some instances it may be necessary to include local excursions of the surface from the mean in the derivation of expressions for field strengths both above and internal to the conductor. Even slight boundary irregularities will perturb the electromagnetic field within the conductor from the value corresponding to a smooth surface. However, the comparative degree to which the fields are influenced by boundary roughness in various frequency ranges for a given conducting dielectric is a question which often remains to be answered.

1 This work was supported by the Office of Naval Research under Contract Nonr $3185(00)$

${ }_{2}$ The author's present address is Boeing Scientific Research Laboratories, Geo-astrophysies Laboratory, The Boeing Co., Seattle, Wash.
A preliminary study of this problem has been reported by Wait [1959b] who considered a uniform plane wave in free space traveling in a direction parallel to the mean (plane) boundary of the conducting dielectric. The interface was taken to be a sinusoidal wave form with an amplitude much smaller than the period, with the magnitude of both being much less than the free space wavelength. Wait found that the resulting perturbation of the field within the conductor was proportional to the amplitude of the wavy surface in the first approximation.

The present investigation is concerned with the propagation of electromagnetic waves within a conducting medium bounded by a rough interface in the presence of a prescribed current source. The precise configuration of the surface, however, is presumed to be unknown, with only its statistical properties being specified. As in the study performed by Wait, it is assumed that the vertical and horizontal scales of the surface irregularities are very small compared with the free space wavelength. A related problem of practical interest is the question of very low frequency signal propagation into the sea; for this reason, the conducting medium is referred to as the sea throughout the discussion. The general approach outlined here is applicable to other physical situations as well, provided certain relationships among the various parameters of importance are satisfied.

For the low frequencies under consideration, sea water is characterized by an electrical conductivity 
$\sigma_{s}$ of about 4 mhos per meter and a relative permittivity $\epsilon_{s}$ of about 80 . Conduction currents are much larger than displacement currents, and, as a result, the expression for the relative index of refraction $N$ of the sea can be simplified to read

$$
N=\left(\epsilon_{s}+i \frac{\sigma_{s}}{\omega \epsilon_{0}}\right)^{1 / 2} \doteq\left(\frac{\sigma_{s}}{\omega \epsilon_{0}}\right)^{1 / 2} \exp \left(i \frac{\pi}{4}\right),
$$

where the time dependence of all field quantities is $\exp (-i \omega t)$. Cosequently, the effective propagation constant $\beta$ can be written in the approximate form

$$
\beta=k_{0} N=\beta_{0} \exp \left(i \frac{\pi}{4}\right)
$$

where

$$
\beta_{0} \doteq k_{0}\left(\frac{\sigma_{s}}{\omega \epsilon_{0}}\right)^{1 / 2} \text {. }
$$

In terms of $\beta_{0}$, the effective wavelength $\lambda_{s}$ in the sea and the skin depth $\delta_{s}$ can be expressed as

and

$$
\lambda_{s}=2 \pi \sqrt{2} \beta_{0}^{-1}=1580 / \sqrt{f_{0}} \mathrm{~m}
$$

$$
\delta_{s}=\sqrt{2} \beta_{0}^{-1}=252 / \sqrt{f_{0}} \mathrm{~m} .
$$

Since the magnitudes of these parameters are of some importance to the developments which follow, plots are presented in figure 1 of $\lambda_{s}$ and $\delta_{s}$ together with the free space wavelength $\lambda_{0}$ as functions of frequency $f_{0}$ over a wide frequency range.

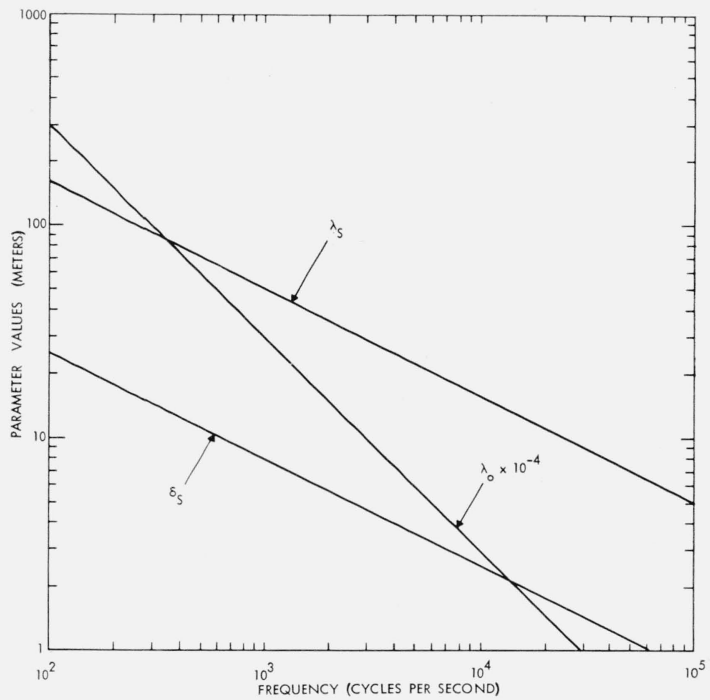

Figure 1. Variation of $\lambda_{\mathrm{s}}, \delta_{\mathrm{s}}$ and $\lambda_{\mathrm{o}}$ with frequency.

The geometry of the problem is illustrated in figure 2. A point source is located on the positive $z_{0}$-axis and the observer is situated at the position $\left(\rho_{0}, \theta_{0},-\left|z_{0}\right|\right)$. It will be assumed that the roughness of the ocean surface is unidimensional in character with the waves running in a direction which forms an angle $\phi$ with the $x_{0}$-axis. It will be convenient at a later stage in the analysis to employ a coordinate system whose origin is directly above the observer (at $\rho_{0}, \theta_{0}, 0$ ) with the $x$-axis parallel to the wave direction. In this system the local height $h$ of the ocean surface is expressible as a function of $x$ alone. (The planes $z=0$ and $z_{0}=0$ are coincident with the mean surface of the sea.)

The electromagnetic field (or, alternatively, the Hertz potential) induced by a point source in the presence of a semi-infinite conducting dielectric with a smooth interface is usually given in a component form appropriate to a cylindrical coordinate system. In the present work, however, we shall be interested primarily in horizontal subsurface electric field components $E_{x s}$ and $E_{y s}$; i.e., the components in directions parallel to and perpendicular to the wave direction, respectively. In the process of deriving expressions for these fields we shall have occasion to utilize the $x, y$, and $z$ components of the free space electric field appropriate to the smooth surface problem. Referring to figure 2 , the transformation from cylindrical components $\left(E_{\rho_{0}}, E_{q_{0}}, E_{z_{0}}\right)$ to Cartesian components $\left(E_{x}, E_{y}, E_{z}\right)$ is simply

$$
\begin{aligned}
& E_{x}=E_{\rho_{0}} \cos \left(\theta_{0}-\phi\right)-E_{\theta_{0}} \sin \left(\theta_{0}-\phi\right) \\
& E_{y}=E_{\rho_{0}} \sin \left(\theta_{0}-\phi\right)+E_{\theta_{0}} \cos \left(\theta_{0}-\phi\right) \\
& E_{z}=E_{z_{0}} .
\end{aligned}
$$

In section 2 approximate integral expressions are derived for the $x$ and $y$ components of the electric field within a moderately rough sea, in terms of the field $\left(E_{x}, E_{y}, E_{z}\right)$ in free space induced by an arbitrary point source above a smooth sea. Subsequent to the introduction in section 3 of certain elementary assumptions regarding the statistics of the surface irregularities a quantity is derived which provides a measure of the effect of surface roughness upon the amplitude of the subsurface signal. Finally, in section 4 , a specific source is assumed ; namely, a vertical electric dipole positioned at $\left(x_{0}, y_{0}, z_{0}\right)=(0,0,0+)$, transmitting at a frequency $f_{0}$ in the VLF range.

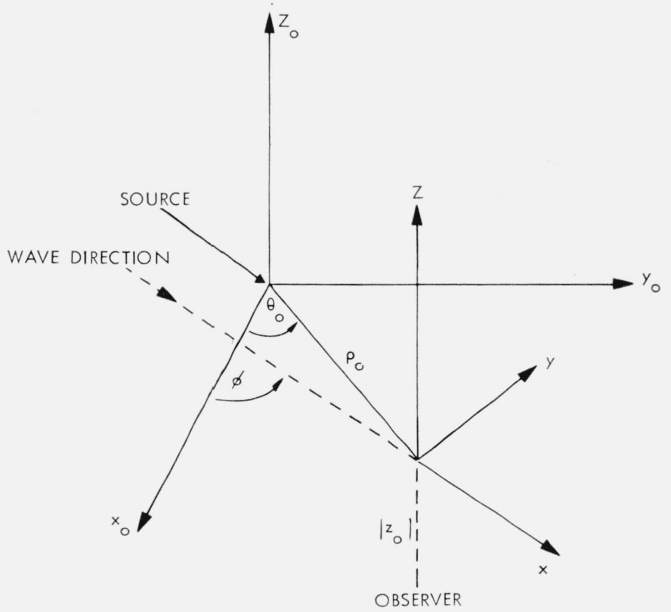

FIGURE 2. Geometry of the problem of propagation into $a$ rough sea. 
Quantitative descriptions of the amplitude fluctuations of $E_{x s}$ (the component of the subsurface electric field in the wave direction) are presented for varying degrees of surface roughness and various observer locations.

\section{Horizontal Electric Field Within the Rough Sea}

The electric field within the sea can be obtained by direct integration of Maxwell's equations using the method of Stratton and Chu [1941], modified slightly to include the finite conductivity of the medium. It should be remarked that the derivation presented by these authors assumes a time dependence of the form $\exp (-i \omega t)$ whereas, in our case, the changing structure of the sea surface produces a more complex temporal variation. For frequencies greater than a few tens of cycles per second, however, the intrinsic period of the electromagnetic fields is far in excess of periods characterizing changes in the sea surface profile. Consequently, the integration of the field equations can be carried out with a harmonic time dependence as a "quasi-steady state" approximation which applies when the ocean surface profile is a slowly varying function of time. It will be understood in the sequel that the local deviation $h$ of the surface from the mean plane $z=0$ is a slowly varying function of time without explicitly exhibiting its dependence upon $t$.

We shall employ the nondimensional variables:

$$
\begin{aligned}
& x^{\prime}=x /\left|z_{0}\right| \\
& y^{\prime}=y /\left|z_{0}\right| \\
& z^{\prime}=z /\left|z_{0}\right| \\
& h^{\prime}=h /\left|z_{0}\right| \\
& R^{\prime}=R /\left|z_{0}\right|=\sqrt{\left(x^{2}+y^{2}\right) /\left|z_{0}^{2}\right|} \\
& \mathbf{r}^{\prime}=-x^{\prime} \hat{\mathbf{x}}-y^{\prime} \hat{\mathbf{y}}-\left(1+h^{\prime}\right) \hat{\mathbf{z}}
\end{aligned}
$$

where $\mathbf{r}^{\prime}$ is the (normalized) vector from the point of integration to the observer. Moreover, the true vertical and horizontal scales of ocean surface roughness, $\sigma_{0}$ and $\delta_{0}$, will be written as

$$
\sigma_{0}=\sigma\left|z_{0}\right|, \quad \delta_{0}=\delta\left|z_{0}\right| .
$$

As a consequence of the nondimensionalization process, it will be convenient to employ the quantity

$$
B=B_{0} e^{i \frac{\pi}{4}}=\beta\left|z_{0}\right|
$$

in place of $\beta\left|z_{0}\right|$ which appears throughout the analysis.

Omitting the primary source terms, an expression can readily be derived for the electric field $\mathbf{E}_{s}$ at the position of the observer in terms of the electromagnetic field vectors $\left(\mathbf{E}^{-}, \mathbf{H}^{-}\right)$just below the ocean surface. Dropping the primes from the nondimensional variables defined in (7) the modified StrattonChu integral can be written in the form

$$
\begin{aligned}
& \mathbf{E}_{s}=-\frac{1}{4 \pi} \int_{S}\left[i \omega \mu_{0}\left|z_{0}\right|\left(\mathbf{n} \times \mathbf{H}^{-}\right) G\right. \\
&\left.\quad \boldsymbol{\nabla} G \times\left(\mathbf{n} \times \mathbf{E}^{-}\right)+\left(\mathbf{n} \cdot \mathbf{E}^{-}\right) \boldsymbol{\nabla} G\right] d S .
\end{aligned}
$$

In this equation, $G$ is the solution of the threedimensional Helmholtz equation appropriate to a medium characterized by a propagation constant $B /\left|z_{0}\right|$ and with a delta function source term:

$$
G=\frac{\exp (i B r)}{r} .
$$

It is easily shown that

$$
\nabla G=\left(\frac{1}{r^{2}}-\frac{i B}{r}\right) G r .
$$

The $x$-derivative of the local surface height $h$ will be denoted by the symbol $\gamma$. The local normal $\mathbf{n}$ to the surface can thus be written in the form (in the case of unidimensional roughness)

$$
\mathbf{n}=(-\gamma \hat{\mathbf{x}}+\hat{\mathbf{z}})\left(1+\gamma^{2}\right)^{-1 / 2} .
$$

The normal is directed outward from the medium, in the direction of free space.

Certain simplifications can be effected by first utilizing the standard conditions of continuity of the tangential components of $\mathbf{E}^{-}$and $\mathbf{H}^{-}$together with the conservation of charge flow across the boundary :

$$
\begin{aligned}
\mathbf{n} \times \mathbf{E}^{-} & =\mathbf{n} \times \mathbf{E} \\
\mathbf{n} \times \mathbf{H}^{-} & =\mathbf{n} \times \mathbf{H} \\
\mathbf{n} \cdot \mathbf{E}^{-} & =\frac{1}{N^{2}} \mathbf{n} \cdot \mathbf{E}
\end{aligned}
$$

where $\mathbf{E}$ and $\mathbf{H}$ are the electric and magnetic fields just above the surface $S$. Incorporating (11a), (11b), and (13) into (10), the expression for the electric field at the position of the observer appears in the form

$$
\begin{array}{r}
\mathbf{E}_{s}=\frac{1}{4 \pi} \int_{S}\left\{i \omega \mu_{0}\left|z_{0}\right|(\mathbf{n} \times \mathbf{H})-\left(\frac{1}{r^{2}}-\frac{i B}{r}\right)[\mathbf{r} \times(\mathbf{n} \times \mathbf{E})\right. \\
\left.\left.-\frac{1}{N^{2}}(\mathbf{n} \cdot \mathbf{E}) r\right]\right\} \frac{e^{i B r}}{r} d S
\end{array}
$$

In order to extract quantitative information from (14) regarding the effect of a randomly rough ocean surface on the propagated signal, it is necessary to reduce the integrand to a combination of known deterministic functions and stochastic functionals of the surface height $h$. To this end, we place the following restrictions on our considerations:

(A) The sea surface profile is only moderately rough in the sense that the vertical scale $\sigma$ of the irregularity is at least an order of magnitude smaller 
than the horizontal scale $\delta$ (alternatively, the quantity $\gamma$ is to be considered at least an order of magnitude less than unity); at frequencies in the VLF range and lower, the scales of the surface irregularities are much smaller than the free space wavelength;

(B) The horizontal displacement $\rho_{0}$ of the observer from the primary source and the free space wavelength $\lambda_{0}$ are much greater than the skin depth $\delta_{s}$; consequently, the horizontal variation in amplitude and phase of the free space field ${ }^{3}$ about $x=0, y=0$ over distances a few skin depths in extent is negligible;

(C) The location of the observer is such that the local excursions of the ocean surface from the mean can be considered small compared with $\rho_{0}$ and $\left|z_{0}\right|$;

(D) The depth of the observer is somewhat greater than a few $\delta_{s}$ so that $B_{0}^{-1}$ is a small quantity compared with unity; and

(E) The primary source frequency is sufficiently low to insure the smallness of $1 /|N|$.

The upper limit of the frequency range is determined in part by restriction (E) and in part by the requirement that the approximation expressed by (1) be valid. On the other hand, restriction (D) implies a lower limit on $f_{0}$ which depends upon the magnitude of $\left|z_{0}\right|$. For example, for depths of the order of $50 \mathrm{~m}$, the transmitter frequency should be greater than about $1 \mathrm{kc} / \mathrm{s}$. When $\left|z_{0}\right|$ is considerably greater than this, the minimum frequency is correspondingly lower, extending to a few tens of cycles per second for depths in excess of $200 \mathrm{~m}$ or so. We also remark that the factor exp $(i B r)$ in (14) provides for exponential damping of contributions to the integral from portions of the integrand evaluated at $x$ and $y$ in excess of a few $\delta_{s} /\left|z_{0}\right|$. This last observation, together with foregoing restrictions, implies that the quantities $B_{0}^{-1}, h, \gamma, R^{2}$ and $N^{-1}$ can all be regarded as at least first order in smallness.

It is natural, therefore, to perform expansions within the integrand of (14) in series involving powers of the smallness parameters. For example, the inverse of the distance $r$ has the expansion

$$
\begin{aligned}
r^{-1}=(1+ & \left.2 h+h^{2}+R^{2}\right)^{-1 / 2} \\
& \doteq 1-\frac{1}{2} R^{2}+\frac{3}{8} R^{4}-h+\frac{3}{2} h R^{2}+h^{2}+\ldots
\end{aligned}
$$

where terms of lower order than the second have been omitted. Simplification of the remaining inverse powers of $r$ proceeds in the same manner. The factor exp $(i B r)$ may be expressed approximately by retaining terms up to the first order in the expansion for $r$ and then performing the substitution, giving

$$
\exp (i B r)=\exp \left[i B\left(1+\frac{1}{2} R^{2}+h\right)\right] \text {. }
$$

${ }^{3}$ In most cases of practical interest, the expression for the field at a height $z$ involves quantities such as $k_{0} \rho_{0}, \boldsymbol{\beta} \rho_{0}$, and trigonometric functions of $\theta_{0}$. The inequalities of restriction (B) insure the slow variation of the expression when $\rho_{0}$ and $\rho_{0} \theta_{0}$ are altered by a few $\delta_{s}$. An inspection of figure 1 reveals that the inequalities are satisfied for $\rho_{0}$ in excess of a few hundred meters over the frequency range of interest here.
Correct to all orders, the normally directed differential element of area $\mathbf{n} d S$ can be written as

$$
\mathbf{n} d S=(-\gamma \hat{\mathbf{x}}+\hat{\mathbf{z}}) d x d y .
$$

Moreover, we observe that according to restriction (B) the variation of the free space electric field in the horizontal direction is small throughout a region centered at $(x, y)=(0,0)$ and a few $\delta_{s}$ in extent. This suggests that $\mathbf{E}(x, y, z=h)$ can be replaced approximately by $\mathbf{E}(0,0, z=h)$ which, in turn, can be expanded in the form

$$
\mathbf{E}(0,0, z=h) \equiv \boldsymbol{\phi}(h)=\boldsymbol{\phi}(0)+h \boldsymbol{\phi}^{\prime}(0)+\frac{1}{2} h^{2} \boldsymbol{\phi}^{\prime \prime}(0)+\ldots,
$$

where the prime indicates differentiation with respect to z. A similar approximation holds for the magnetic field. Since the roughness scales of the sea surface are extremely small compared with the wavelength, the electromagnetic fields in free space are scarcely altered by the surface irregularities. Hence, the derivatives of $\boldsymbol{\phi}(z)$ can be calculated from the solution to the smooth sea problem with little error and the final result is a representation for $\mathbf{E}$ for substitution into (14) in which the only variable is $h$. The expression for the magnetic field is treated in the same fashion.

The approximate expression for $\mathbf{E}_{s}$ is obtained by first substituting (15), (16), (17), and (18) into (14), together with the appropriate expansions of higher inverse powers of $r$ and the series development of $\mathbf{H}$ which is the counterpart of (18). The final result for $\mathbf{E}_{s}$ will be correct to the second order if those terms of the integrand are dropped which involve powers of smallness parameters which are lower than second order.

The $x$ - and $y$-components of the subsurface electric field are of principal interest here and can be written in the form (correct to the second order) of integrals over all $x-y$ space in the manner just described. Since the random variable $h$ depends only upon $x$, the integration over $y$ can be performed at once. Some compactness of notation is achieved by replacing $\mathbf{H}$ with

$$
\psi=\frac{\omega \mu_{0}}{\beta} \mathbf{H}
$$

Omitting the algebraic details, the final result for the horizontal components of the subsurface electric field, following integration over $y$, can be expressed as

$$
\begin{aligned}
& E_{j s}=\frac{1}{2}\left(\frac{B_{0}}{2 \pi}\right)^{1 / 2} e^{i\left(B-\frac{\pi}{8}\right)} \sum_{k=1}^{11} \\
& A_{j k} \int_{-\infty}^{\infty} e^{i \frac{B}{2} x^{2}} e^{i B h} f_{k}(x, h, \gamma) d x,
\end{aligned}
$$

where $j=x$ or $y$, and 


$$
\begin{array}{lll}
f_{1}=1 & f_{5}=\gamma & f_{9}=h\left(x^{2}+i B^{-1}\right) \\
f_{2}=x & f_{6}=x \gamma & f_{10}=\gamma\left(x^{2}+i B^{-1}\right) \\
f_{3}=x^{2}+i B^{-1} & f_{7}=h^{2} & f_{11}=x^{4}+2 i B^{-1} x^{2}-3 B^{-2} \\
f_{4}=h & f_{8}=h \gamma &
\end{array}
$$

and

$$
\begin{aligned}
& A_{x 1}=-\psi_{y}+\left(1+i B^{-1}\right) \phi_{x} \\
& A_{x 2}=N^{-2} \phi_{z} \\
& A_{x 3}=\frac{1}{2} \psi_{y}-\left(1+\frac{3}{2} i B^{-1}\right) \phi_{x} \\
& A_{x 4}=\psi_{y}-\psi_{y}^{\prime}-\left(1+2 i B^{-1}\right) \phi_{x}+\left(1+i B^{-1}\right) \phi_{x}^{\prime} \\
& A_{x 5}=\left(1+i B^{-1}\right) \phi_{z} \\
& A_{x s}=0 \\
& A_{x 7}=-\psi_{y}+\psi_{y}^{\prime}-\frac{1}{2} \psi_{y}^{\prime \prime}+\phi_{x}-\phi_{x}^{\prime}+\frac{1}{2} \phi_{x}^{\prime \prime} \\
& A_{x 8}=-\phi_{z}+\phi_{z}^{\prime} \\
& A_{x 9}=-\frac{3}{2} \psi_{y}+\frac{1}{2} \psi_{y}^{\prime}+3 \phi_{x}-\phi_{x}^{\prime} \\
& A_{x 10}=-\phi_{z} \\
& A_{x 11}=\phi_{x}-\frac{3}{8} \psi_{y} \\
& A_{y 1}=\psi_{x}+\left(1+i B^{-1}\right) \phi_{y} \\
& A_{y 2}=0 \\
& A_{y 3}=-\frac{1}{2} \psi_{x}-\left(1+\frac{3}{2} i B^{-1}\right) \phi_{y} \\
& A_{y 4}=-\psi_{x}+\psi_{x}^{\prime}-\left(1+2 i B^{-1}\right) \phi_{y}+\left(1+i B^{-1}\right) \phi_{y}^{\prime} \\
& A_{y 5}=\psi_{z} \\
& A_{y 6}=-\left(1+i B^{-1}\right) \phi_{y} \\
& A_{y 7}=\psi_{x}-\psi_{x}^{\prime}+\frac{1}{2} \psi_{x}^{\prime \prime}+\phi_{y}-\phi_{y}^{\prime}+\frac{1}{2} \phi_{y}^{\prime \prime} \\
& A_{y 8}=-\psi_{z}+\psi_{z}^{\prime} \\
& A_{y 9}=\frac{3}{2} \psi_{x}-\frac{1}{2} \psi_{x}^{\prime}+3 \phi-\phi_{y y}^{\prime} \\
& A_{y 10}=-\frac{1}{2} \psi_{z} \\
& A_{y 11}=\phi_{y}+\frac{3}{8} \psi_{x} .
\end{aligned}
$$

In these expressions $\phi_{j}^{(n)}$ and $\psi_{j}^{(n)}$ are understood to mean $\phi_{j}^{(n)}(0)$ and $\psi_{j}^{(n)}(0)$ where the differentiation precedes specialization of the argument.

\section{Sea Surface Structure and Electric Field Amplitude Fluctuation}

Field components $E_{x s}$ and $E_{y s}$ resulting from a particular wave structure could now be calculated simply by substituting the appropriate function for $h$ into (20) and performing the remaining integrations numerically or in some other approximate way. In the present study the more general assumption is made that the sea profile is a stochastic function of $x$. A quantitative measure of the effect of boundary irregularity on the subsurface field can be obtained by calculating the mean square deviation (msd) of the field amplitude from its average value. Denoting mathematical (ensemble) averages (or expectations) by $\mathscr{E}\{\}$ we have (after normalization)

$$
\begin{aligned}
\operatorname{msd}\left(\left|E_{j s}\right|\right)= & \mathscr{E}\left\{[ E _ { j s } - \mathscr { E } \{ E _ { j s } \} ] \left[E_{j s}^{*}\right.\right. \\
& \left.\left.\quad-\mathscr{E}^{*}\left\{E_{j s}\right\}\right]\right\} / \mathscr{E}\left\{E_{j s}\right\} \mathscr{E}^{*}\left\{E_{j s}\right\} \\
= & {\left[\mathscr{E}\left\{E_{j s} E_{j s}^{*}\right\} / \mathscr{E}\left\{E_{j s}\right\} \mathscr{E}^{*}\left\{E_{j s}\right\}\right]-1 . }
\end{aligned}
$$

However, the analysis can proceed only after some assumptions are made concerning the statistics of the surface profile. Therefore, an elementary statistical model is introduced and certain averages necessary to the calculation of (21) are derived.

The simplest classical model of ocean waves consists of an infinite succession of one-dimensional periodic progressive waves of nearly equal amplitude. In actuality, sea wave structure is almost never characterized by a single crest height or crest-to-crest distance. Instead, the profile of the surface throughout any local region is more realistically described as a superposition of a great many waves with a wide range of amplitudes and wavelengths. In the presence of moderate or high winds, the complexity of the wave configuration is enhanced and the surface loses nearly all appearance of regularity. The local departure of the sea surface from its mean value is therefore treated as a random variable in the present study.

Sea surface structure is an extensive subject in itself and cannot be presented in any detail here (for an excellent summary the reader is referred to Defant [1961]). Suffice it to say that the statistics of ocean wave structure is dependent upon a great number of factors (including wind speed, duration, and fetch) and that the precise nature of this dependence is not thoroughly understood at the present time. For this reason (and for the sake of comparative mathematical simplicity) the surface height $h$ will be described as a stationary Gaussian random process with a constant dispersion $\sigma^{2}$. The surface height has been assumed to vary with a single linear coordinate since swells and seas of moderate roughness often exhibit a general tendency toward unidimensional structure over fairly extensive regions.

The restriction to "moderate" surface roughness, together with the assumption that $\gamma=\frac{d h}{d x}$ is a small quantity (for most realizations), requires that the variance (the expectation of the product $h\left(x_{1}\right) h\left(x_{2}\right)$ ) imply a value for the mean square local surface slope which is second order in smallness. It will be assumed here that the variance is a Gaussian function of the form

$$
\mathcal{E}\left\{h_{1} h_{2}\right\}=\sigma^{2} \rho\left(x_{1}, x_{2}\right)=\sigma^{2} \exp \left[-\left(x_{2}-x_{1}\right)^{2} / \delta^{2}\right]
$$


where the subscript affixed to $h$ signifies the point at which it is to be evaluated. It can be shown (see section 6, appendix A) that the mean square surface slope is given by

$$
\mathscr{E}\left\{\gamma^{2}\right\}=\lim _{x_{2} \rightarrow x_{1}} \mathscr{E}\left\{\gamma_{1} \gamma_{2}\right\}=\lim _{x_{2} \rightarrow x_{1}} \sigma^{2} \frac{\partial^{2} \rho}{\partial x_{1} \partial x_{2}}=2 \frac{\sigma^{2}}{\delta^{2}} .
$$

As $\sigma$ and $\delta$ have been defined in this section, they are measures of boundary roughness in the vertical and horizontal directions and can be considered identical to the roughness scales previously employed. Hence, in accordance with the discussion in section 2 , the mean square slope is a second order quantity with the variance defined as in $(22)$. It should be noted that if an exponential variance were assumed, this condition would not be satisfied since the equalities in (23) would lead to an infinite value for the mean square slope.

The calculation of the mean square deviation of electric field amplitude requires the expectations of both $E_{j s}$ and the product $E_{j s} E_{j s}^{*}$. For the former quantity we have, from (20),

$$
\begin{aligned}
\mathscr{E}\left\{E_{j s}\right\}= & \frac{1}{2} \sqrt{\frac{\overline{B_{0}}}{2 \pi}} e^{i\left(B-\frac{\pi}{8}\right)} \sum_{m} \\
& A_{j m} \int_{-\infty}^{\infty} e^{i \frac{B}{2} x^{2}} \mathscr{E}\left\{e^{i B h} f_{m}(x, h, \gamma)\right\} d x
\end{aligned}
$$

and for the ensemble average of $E_{j s} E_{j s}^{*}$, we find

$$
\begin{aligned}
& \mathscr{E}\left\{E_{j s} E_{j s}^{*}\right\}=\frac{B_{0}}{8 \pi} e^{-\sqrt{2} B_{0}} \sum_{m} \sum_{n} \\
& A_{j m} A_{j n}^{*} \int_{-8}^{\infty} \int_{-} e^{i \frac{B_{1}}{2} x_{1}^{2}-i \frac{B_{2}}{2} x_{2}^{2}} \cdot \mathscr{E}\left\{e^{i B_{1} h_{1}-i B_{2} h_{2}}\right. \\
&\left.f_{m}\left(x_{1}, h_{1}, \gamma_{1}\right) f_{n}^{*}\left(x_{2}, h_{2}, \gamma_{2}\right)\right\} d x_{1} d x_{2},
\end{aligned}
$$

where we have introduced the notation

$$
\begin{aligned}
& B_{1}=B \\
& B_{2}=B^{*} .
\end{aligned}
$$

Since we are interested only in results correct to the second order, it is permissible to neglect lower order terms (such as $m=8, n=4)$ in the double sum in (25).

The various expectations appearing in the integrands of (24) and (25) are readily calculated with the aid of the Karhunen representation (see e.g., Davenport and Root [1958]). The final results for those expectations required in the calculation of (24) and of (25), subsequent to the deletion of terms which are lower than second order, are given in section 6 , appendix A. It should be noted that the off-diagonal terms of the Cauchy product of $E_{j s}$ and $E_{j s}^{*}$ occur in complex conjugate pairs so that only one term of each pair need be calculated.

With the aid of the equations listed in appendix A, the definitions following (20), and the relations given in (19), it is easily shown that the expectations in (24) have the simple form

$$
\mathscr{E}\left\{e^{i B h} f_{m}(x, h, \gamma)\right\}=e^{-\frac{i}{2} b} F_{m}(x)
$$

where

$$
b=B_{0}^{2} \sigma^{2}
$$

and where $F_{m}(x)$ is a simple polynomial in $x$ with complex coefficients. The integration over $x$ can be performed at once, with the result

$$
\begin{aligned}
\mathscr{E}\left\{E_{j 0}\right\}= & \frac{1}{2} e^{-\frac{\sqrt{2}}{2} B_{0}} e^{i\left(\frac{\sqrt{2}}{2} B_{0}-\frac{b}{2}\right)} \\
& {\left[A_{j 1}+2 \frac{i}{B} A_{j 3}+i B \sigma^{2} A_{j 4}\right.} \\
+ & \left.\sigma^{2}\left(1-B^{2} \sigma^{2}\right) A_{j 7}-2 \sigma^{2} A_{j 9}-\frac{8}{B^{2}} A_{j 11}\right] .
\end{aligned}
$$

The expectations appearing in (25) are somewhat more complicated; utilizing (19), (20), (22), and (26), as well as equations in section 6 , appendix $A$, we find that

$$
\begin{aligned}
\mathscr{E}\left\{e^{i B_{1} h_{1}-i B_{2} h_{2}} f_{m}\left(x_{1}, h_{1}, \gamma_{1}\right) f_{n}^{*}\left(x_{2}, h_{2}, \gamma_{2}\right)\right\} \\
=e^{b \exp \left[-\left(x_{2}-x_{1}\right)^{2} / \delta^{2}\right]} F_{m n}\left(x_{1}, x_{2}\right)
\end{aligned}
$$

where $F_{m n}\left(x_{1}, x_{2}\right)$ is a polynomial in $x_{1}$ and $x_{2}$. Thus, the calculation of (25) has been reduced essentially to the problem of evaluating the integral

$$
\begin{array}{r}
\iint_{-\infty}^{\infty} e^{i \frac{B_{1}}{2} x_{1}^{2}-i \frac{B_{2}}{2} x_{2}^{2}} e^{b \exp \left[-\left(x_{2}-x_{1}\right)^{2 /} \delta^{2}\right]} \\
F_{m n}\left(x_{1}, x_{2}\right) d x_{1} d x_{2}=I_{m n}, \text { say. }
\end{array}
$$

First making the change of variable

$$
\begin{aligned}
& x_{1}=\frac{\delta}{\sqrt{2}}\left(\frac{u}{\sqrt{a}}-e^{i \frac{\pi}{4}} v\right) \\
& x_{2}=\frac{\delta}{\sqrt{2}}\left(\frac{u}{\sqrt{a}}+e^{-i \frac{\pi}{4}} v\right)
\end{aligned}
$$

and substituting into (31), we have

$$
I_{m n}=\frac{\delta^{2}}{\sqrt{2 a}} \iint_{-\infty}^{\infty} e^{-u^{2}-a v^{2}} e^{b e^{-v^{2}}} F_{m n}(u, v) d u d v
$$

where

$$
a=\frac{\sqrt{2} B_{0} \delta^{2}}{4} .
$$

For realistic values of $\sigma$ (less than about 3 meters), the parameter $b=B_{0}^{2} \sigma^{2}$ is less than unity for the range of frequencies of interest here. Consequently, it is possible to expand the second exponential in (33) to obtain 


$$
I_{m n}=\frac{\delta^{2}}{\sqrt{2 a}} \sum_{l=0}^{\infty} \frac{b^{l}}{l !} \int_{-\infty}^{\infty} \int_{\infty} e^{-u^{2}} e^{-(a+l) v^{2}} F_{m n}(u, v) d u d v .
$$

Since $F_{m n}(u, v)$ is a polynomial in $u$ and $v$, it is a simple matter to calculate $I_{m n}$. The final result for the expectation of $E_{j s} E_{j s}^{*}$ is obtained by substituting the functions $I_{m n}$ into $(25)$. This result, together with (29), provides all that is required for the calculation of (21). The complete expression for the normalized mean square deviation of $\left|E_{j s}\right|$ is recorded in section 6, appendix B. In the next section a specific source is assumed and numerical results are presented for various frequencies, sea states, and observer locations.

\section{Numerical Results for a Vertical Electric Dipole}

An application of the foregoing developments which may be of some practical interest is the case of a vertical electric dipole on the surface of the sea, transmitting at very low frequencies. It will be supposed that the observer's location corresponds to a horizontal displacement $\rho_{0}$ which is several free space wavelengths in extent. The depth $\left|z_{0}\right|$ of the observer below the mean ocean surface is assumed sufficient to insure the smallness of $B_{0}{ }^{-1}$.

The quantities of interest here are the mean square amplitude fluctuations of the horizontal components of the subsurface electric fields due to sea surface irregularities. Although at low radiofrequencies the wavelength in free space is enormously large compared with the scales $\sigma_{0}$ and $\delta_{0}$ and the free space fields are hardly perturbed at all, we nevertheless expect the surface structure to produce a pronounced effect upon the amplitudes of the subsurface fields, particularly in the case of a vertical electric dipole. The reason for this can be seen by noting that, in the case of a perfectly smooth boundary, the dominant contribution to $E_{x s}$ and $E_{y s}$ arises from the horizontal component $E_{\rho 0}$ of the free space electric field, which is continuous across the surface $z=0$. In the radiation zone, the $z_{0}$-component of the electric field of a vertical electric dipole on the upper side of the boundary of a conducting dielectric is greater than $E_{\rho 0}$ by a factor of $N$, and this component is reduced by a factor of the order of $1 / N^{2}$ across the boundary of the conductor. On the other hand, when the surface is characterized locally by a nonzero slope, the field $E_{z 0}$ will contribute to the local tangential electric field at the true surface. Since this component is coupled through the surface without change, it can contribute significantly to the horizontal component of the subsurface electric field. In the case of a vertical electric dipole over a unidimensionally rough sea, it is expected that this effect will manifest itself primarily in the $x$-component of the subsurface field. This observation is verified by an inspection of (20), where the contributions to $E_{x s}$ from the projection of $E_{z 0}$ on the true surface are contained in the terms $k=5,8$, and 10 which involve the product $\gamma E_{z}$.
In order to perform numerical calculations, it was first necessary to obtain expansions of the type (18) for the electric and magnetic fields above a smooth conducting dielectric. The expressions used for this purpose were those developed by Norton [1937] for a vertical electric dipole on a conducting half-plane. The coefficients of these expansions are required for calculation of the $A_{j k}$ recorded in (20).

The results of several representative computations are shown in figures $3,4,5$, and 6 . These calculations were performed to assess the effect of varying parameters on the mean square deviation of the amplitude of $E_{x s}$. In figures 3, 4, and 5, the direction of the ocean waves was assumed to be parallel to the radial displacement $\rho_{0}$ of the observer from the dipole. The magnitude of $\rho_{0}$ itself was fixed at $500 \mathrm{~km}$. Figures 3 and 4 reveal a steady decrease in the amplitude fluctuation with increasing depth and with increasing frequency for fixed sea states (constant $\sigma_{0}$ and $\delta_{0}$ ). Figure 5 shows the variation of the fluctuation intensity with increasing correlation distance $\delta_{0}$ for several values of $\sigma_{0}$. In contrast with figures 3 and 4 these curves are not monotonic, but instead indicate a maximum fluctuation level which depends upon the sea state, other parameters being fixed.

Figure 6 shows the effect of varying the angular coordinate $\theta_{0}$ of the observer for fixed sea states when the ocean wave direction is parallel to the $y_{0}$-axis. The steady increase in the relative fluctuation level as the observer moves in the direction of decreasing $\theta_{0}$ is not unexpected. When $\theta_{0}$ is equal to zero, the observer is located on the $x_{0}$-axis. The ocean wave direction is $\phi=90^{\circ}$ and therefore the $x$-axis (and $E_{x s}$ ) is perpendicular to $x_{0}$. The source is a vertical electric dipole and for a smooth sea $E_{x s}$

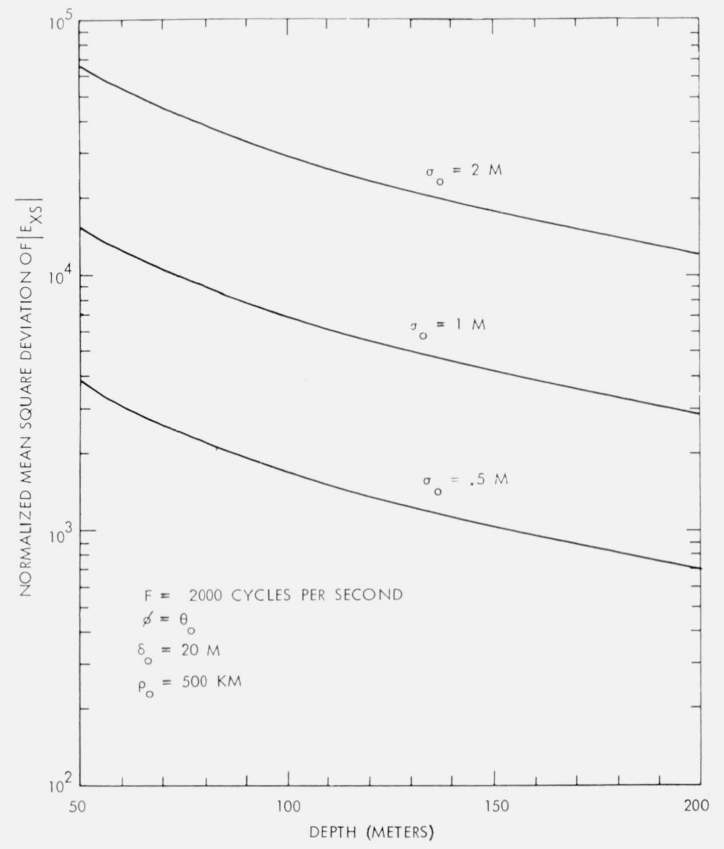

Figure 3. Variation of $m s d\left(\left|\mathrm{E}_{\mathrm{xs}}\right|\right)$ with observer depth. 


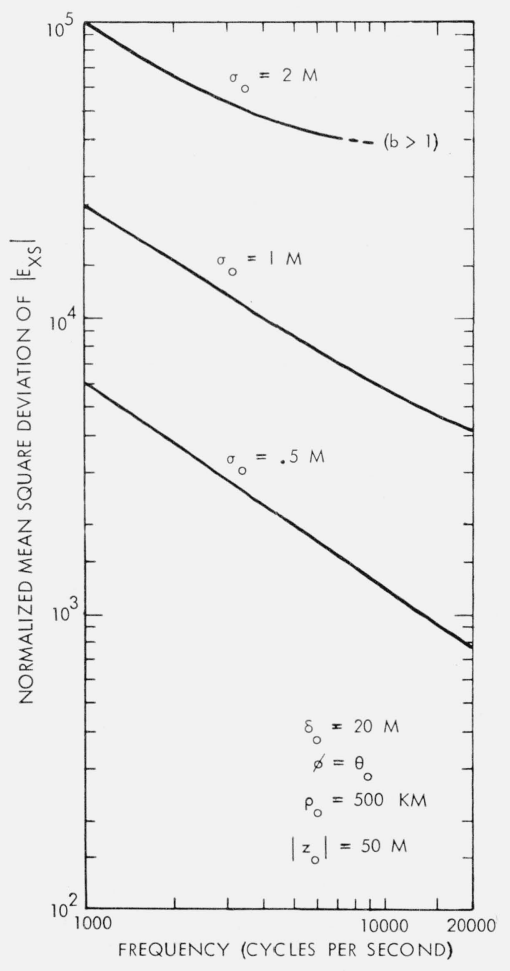

FIGURE 4. Variation of $m s d\left(\left|\mathrm{E}_{\mathbf{x}}\right|\right)$ with transmitter frequency.

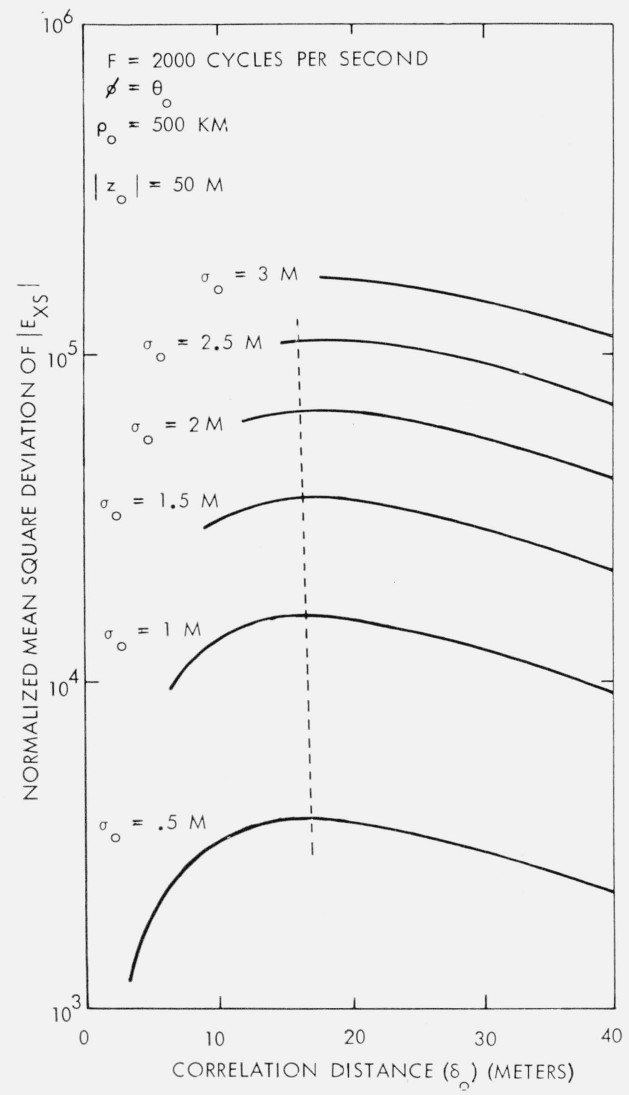

Figure 5. Variation of msd $\left(\left|\mathrm{E}_{\mathrm{xs}}\right|\right)$ with correlation distance.

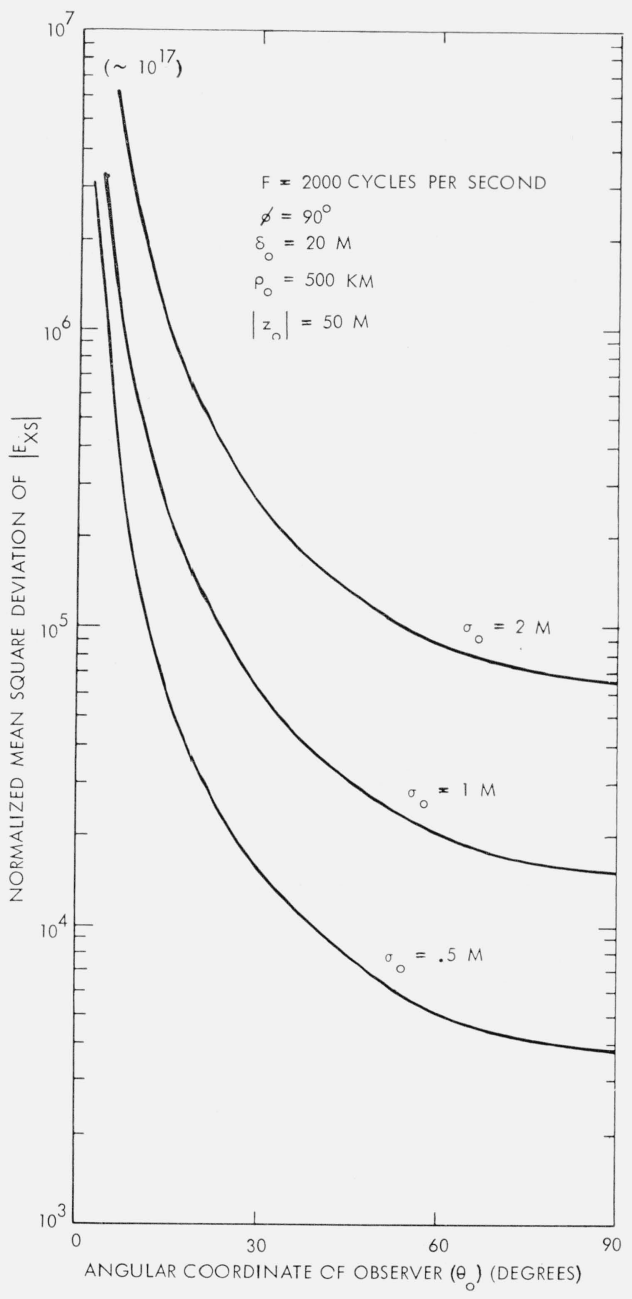

FiguRE 6. Variation of $m s d\left(\left|\mathrm{E}_{\mathrm{x}}\right|\right)$ with angular coordinate of observer.

would be identically zero. In the present instance, however, the subsurface field $E_{x s}$ does not vanish because of the fact that the vertical component of the free space field can in general have a component tangential to the true surface. On the other hand, the mean field strength itself is at a minimum when $\theta_{0}=0^{\circ}$ and thus the relative fluctuation in intensity as defined here is greatest on the $x_{0}$-axis. Calculations were also performed with $\rho_{0}$ as the running variable, but only slight changes in relative fluctuation intensity were predicted.

\section{Physical Interpretation and Conclusions}

A few general observations can be made concerning the influence of ocean surface roughness on subsurface field amplitude fluctuations by returning to figure 5. For a fixed rms surface wave height, the mean square deviation of $\left|E_{x s}\right|$ is seen first to increase to a broad maximum and subsequently decrease as the horizontal scale of the surface roughness is increased. A possible explanation of this functional 
behavior is provided by the considerations described below.

Suppose the dipole to be transmitting at a frequency $f_{0}$ to an observer at a depth $\left|z_{0}\right|$. On the average, the predominant contributions to the received signal arise from a roughly circular region of the mean sea surface. The boundary of this region is defined by the intersection of the mean surface with a cone whose vertex is at the observer's location and whose axis is colinear with the $z_{0}$-axis. The vertex angle $\alpha$ will be defined approximately by the relation

$$
\cos \alpha=\left|z_{0}\right| /\left|z_{1}\right|
$$

where $\left|z_{1}\right|$ is the distance from the observer to those points on the mean surface where the contributions to the field at the observer have fallen off by the factor $e^{-1}$. In the case of a vertical electric dipole and nonnegligible surface slopes, the most important contributions originate in regions of maximum slope where the large vertical component of the free space field can contribute to $E_{x s}$. Taking these contributions to be proportional to those of point sources in an infinite medium for order of magnitude estimates, the distance $z_{1}$ can be defined approximately as the solution of the transcendental equation

$$
e^{-1} \frac{\exp \left(-\left|z_{0}\right| / \delta_{s}\right)}{\left|z_{0}\right|}=\frac{\exp \left(-\left|z_{1}\right| / \delta_{s}\right)}{\left|z_{1}\right|}
$$

where $\delta_{s}$ is the skin depth at frequency $f_{0}$. The radial extent $a_{0}$ of the important contributing region of the sea surface is then

$$
a_{0}=\sqrt{\left|z_{1}\right|^{2}-\left|z_{0}\right|^{2}}
$$

Thus, at a fixed depth and frequency the radius $a_{0}$ is determined.

When $\delta_{0}$ (the horizontal scale of irregularity, corresponding roughly to the length of a surface wave) is sufficiently small compared with $a_{0}$, the surface area of importance will contain a relatively large number of contributing regions, all radiating with more or less random phases. As $\delta_{0}$ decreases, this state of affairs is enhanced and so also is the tendency toward destructive interference at the observer's location, resulting in a net decrease in the relative fluctuation in field intensity. On the other hand, if $\delta_{0}$ is initially large compared with $a_{0}$ and becomes increasingly larger, not only does the probability of finding regions of maximum slope within the area decrease but the excursions of the field at the observer due to these contributions are diminished since they are proportional to the derivative of the local surface profile. Again, the net result is a decrease in relative fluctuation intensity.

Between these two extremes is a transitional state in which, according to the foregoing remarks, the number of important radiating regions lying within the cone is of the order of unity. For the sake of discussion, assume the sea surface to be sinusoidally corrugated with a profile of the form

$$
h=h_{0} \sin \left(2 \pi x / d_{n}\right) .
$$

Then a transitional state in which the fluctuation intensity is a maximum will be similar to the one illustrated in figure 7 where the observer is located below the region of maximum slope and where, for the sake of definiteness, one of the line segments $\left|z_{1}\right|$ (a generator of the cone) is taken to pass through a point in the trough of the wave profile. The

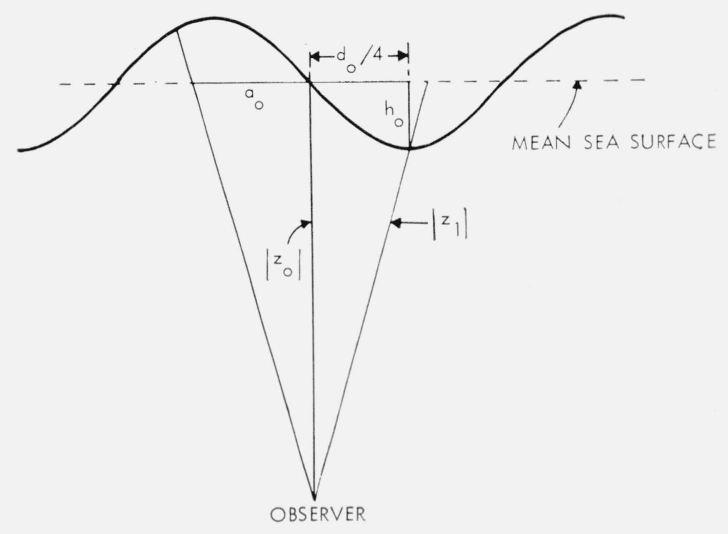

FIGURE 7. Geometry of a representative transitional state.

vertical and horizontal roughness scales $\sigma_{0}$ and $\delta_{0}$ associated with the statistical model can be related to the wave height $h_{0}$ and wavelength $d_{0}$ of the deterministic model by equating the ensemble averages of $h^{2}$ and $\gamma^{2}$ with the mean values of these quantities as obtained from (39). Using (22) and (23) this procedure leads to the relations ${ }^{4}$

$$
\begin{aligned}
& h_{0} \rightarrow \sqrt{2} \sigma_{0} \\
& d_{0} \rightarrow \sqrt{2} \pi \delta_{0} .
\end{aligned}
$$

The validity of the foregoing observations can be tested in a simple manner. The transitional state which is illustrated in figure 7 should correspond roughly to a sea state for which the mean square deviation of $\left|E_{x s}\right|$ is a maximum. The geometry in figure 7 , together with (37), (38), and (40), is sufficient to determine the correlation distance (call it $\delta_{\text {trans }}$ which corresponds to this state when $\sigma_{0}$, $\left|z_{0}\right|$, and $\delta_{s}\left(\right.$ or $\left.f_{0}\right)$ are given. From figure 7 and (40) we have the relations

$$
\delta_{\text {trans }}=\frac{d_{0}}{\sqrt{2} \pi}=\frac{4 a_{0}}{\sqrt{2} \pi\left|z_{0}\right|}\left(\left|z_{0}\right|-h_{0}\right) .
$$

An approximate solution of (37) can be obtained by a single application of Newton's Method. Combining this result with (38) and (41), we obtain

$$
\delta_{\text {trans }}=\frac{4}{\sqrt{2} \pi}\left(\left|z_{0}\right|-\sqrt{2} \sigma_{0}\right)\left\{\left[1+\frac{\delta_{s}(e-1)}{\delta_{s}+e\left|z_{0}\right|}\right]^{2}-1\right\}^{1 / 2}
$$

4 Expressions such as these may be useful in relating statistical parameters to "apparent wave height" and "apparent wavelength" which oceanographers "apparent wave height" and "apparen 
When plots such as those in figure 5 are drawn for several values of $\left|z_{0}\right|$ and $f_{0}$, as well as $\sigma_{0}$, (with $\delta_{0}$ as the running variable) we should expect to find a maximum in each, located at a value of $\delta_{0}$ which corresponds closely with $\delta_{\text {trans }}$ as given by (42). The dashed curve in figure 5 shows the locus of the maxima of curves corresponding to various $\sigma_{0}$ as calculated from (42). However, a test against a single variable is inconclusive. Calculations similar to those in figure 5 were performed for several values of $f_{0}$ and $\left|z_{0}\right|$. Values of $\delta_{\text {trans }}$ were read from these curves and compared with calculations from (42). The results are presented in figures 8 and 9 . The agreement in all three cases is seen to be satisfactory.

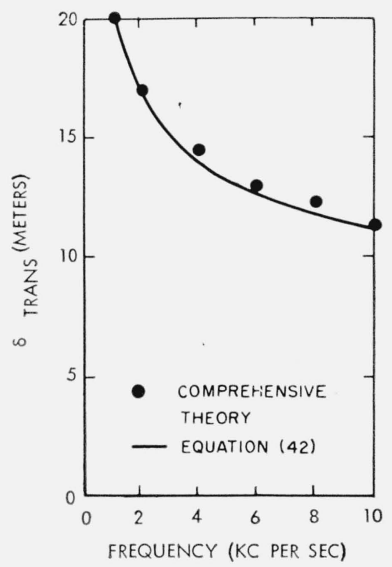

Figure 9. Variation of transitional $\delta$ with observer depth.

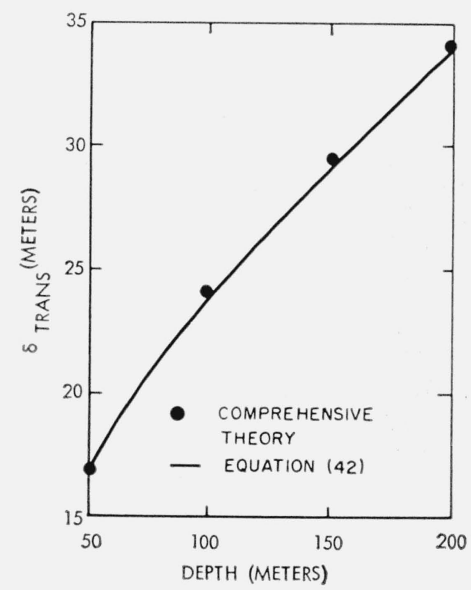

Figure 8. Variation of transitional $\delta$ with frequency.

In conclusion, it should be borne in mind that the quantity selected for evaluation in this paper, i.e., the mean square amplitude deviation of the horizontal components of the subsurface electric field, was chosen primarily to illustrate the method for assessing the effect of boundary roughness on subsurface signals. The same technique can be applied to any component of the electromagnetic field (or combination of components) within the conducting medium. Moreover, the restriction to unidimensional roughness is not essential; bidimensional irregularities can be treated in precisely the same fashion.

The author expresses his thanks to Drs. J. E. Storer and R. V. Row for several helpful discussions of this problem. The assistance of Mr. R. Cassarino who programmed the equations for the computer is also gratefully acknowledged.

\section{Appendix A. Derivation of Certain Statis- tical Averages}

The surface height $h(x)$ is assumed to be a separable random function, continuous for all $x$, with zero mean and variance $\sigma^{2} \rho\left(x_{1}, x_{2}\right)$. In accordance with the Karhunen-Loeve Theorem, there exists an expansion for $h$ of the form

$$
h(x)=\lim \sum \lambda_{p} \phi_{p}(x) z_{p}
$$

in terms of the orthogonal process $z_{p}$ :

$$
\mathcal{E}\left\{z_{p} z_{q}\right\}=\delta_{p q}
$$

where $\delta_{p q}$ is the Kronecker delta and where $\lambda_{p}^{-1}$ and $\phi_{p}(x)$ are the eigenvalues and eigenfunctions of the integral equation

$$
\phi_{p}\left(x_{1}\right)=\lambda_{p}^{-1} \int_{-\infty}^{\infty} \sigma^{2} \rho\left(x_{1}, x_{2}\right) \phi_{p}\left(x_{2}\right) d x_{2} .
$$

An analytical procedure which is closely related to the one discussed by Hoffman [1955] provides us with the auxiliary relations (for a Gaussian Process)

$$
\begin{aligned}
\mathscr{E}\left\{e^{i B_{1} h_{1}-i B_{2} h_{2}}\right\}= & e^{-\frac{\sigma 2}{2}\left(B_{1}^{2}+B_{2}^{2}-2 B_{1} B_{2} \rho\right)} \equiv M\left(B_{1}, B_{2}\right) \\
\mathscr{E}\left\{z_{p} e^{i B_{1} h_{1}-i B_{2} h_{2}}\right\}= & i \lambda_{p}\left[B_{1} \phi_{p}\left(x_{1}\right)-B_{2} \phi_{p}\left(x_{2}\right)\right] M\left(B_{1}, B_{2}\right) \\
\mathscr{E}\left\{z_{p} z_{q} e^{i B_{1} h_{1}-i B_{2} h_{2}}\right\}= & \left\{\delta_{p q}-\lambda_{p} \lambda_{q}\left[B_{1}^{2} \phi_{p}\left(x_{1}\right) \phi_{q}\left(x_{1}\right)\right.\right. \\
& -B_{1} B_{2} \phi_{p}\left(x_{1}\right) \phi_{q}\left(x_{2}\right) \\
& -B_{1} B_{2} \phi_{p}\left(x_{2}\right) \phi_{q}\left(x_{1}\right) \\
& \left.\left.+B_{2}^{2} \phi_{p}\left(x_{2}\right) \phi_{q}\left(x_{2}\right)\right]\right\} M\left(B_{1}, B_{2}\right)
\end{aligned}
$$

These equations, together with the definitions

$$
\begin{aligned}
& \sigma^{2}=\Sigma \lambda_{p}^{2} \phi_{p}^{2}(x) \quad(=\text { const. }) \\
& \sigma^{2} \rho\left(x_{1}, x_{2}\right)=\Sigma \Sigma \lambda_{p} \lambda_{g} \phi_{p}\left(x_{1}\right) \phi_{q}\left(x_{2}\right),
\end{aligned}
$$

are sufficient for the computation of all expectations appearing in (25), subsequent to the deletion of terms of a lower order than the second. Averages 
of the type appearing in (24) can be calculated after specializing $(\mathrm{A}-4)$ by setting $B_{2}$ equal to zero throughout. The various expectations required in the calculation of (24) and (25) are listed below. The results apply when $h$ is a Gaussian process with zero mean and with dispersion and variance as given by $(\mathrm{A}-5)$ :

$\mathscr{E}\left\{e^{i B h}\right\}=e^{-\frac{1}{2} B^{2} \sigma^{2}} \equiv M(B)$

$\mathscr{E}\left\{h e^{i B h}\right\}=i B \sigma^{2} M(B)$

$\mathcal{E}\left\{\gamma e^{i B h}\right\}=0$

$\mathscr{E}\left\{h \gamma e^{i B h}\right\}=0$

$\mathcal{E}\left\{h^{2} e^{i B h}\right\}=\sigma^{2}\left(1-B^{2} \sigma^{2}\right) M(B)$

$\mathcal{E}\left\{e^{i B_{1} h_{1}-i B_{2} h_{2}}\right\}=e^{-\frac{\sigma^{2}}{2}\left(B_{1}^{2}+B_{2}^{2}-2 B_{1} B_{2} \rho\right)} \equiv M\left(B_{1}, B_{2}\right)$

$\mathcal{E}\left\{h_{1} e^{i B_{1} h_{1}-i B_{2} h_{2}}\right\}=i \sigma^{2}\left(B_{1}-B_{2} \rho\right) M\left(B_{1}, B_{2}\right)$

$\mathcal{E}\left\{\gamma_{1} e^{i B_{1} h_{1}-i B_{2} h_{2}}\right\}=-i B_{2} \sigma^{2} \frac{\partial \rho}{\partial x_{1}} M\left(B_{1}, B_{2}\right)$

$\mathcal{E}\left\{h_{1} \gamma_{1} e^{i B_{1} h_{1}-i B_{2} h_{2}}\right\}=B_{2} \sigma^{4} \frac{\partial \rho}{\partial x_{1}}\left(B_{1}-B_{2} \rho\right) M\left(B_{1}, B_{2}\right)$

$\mathscr{E}\left\{h_{2} \gamma_{1} e^{i B_{1} h_{1}-i B_{2} h_{2}}\right\}$

$$
=\sigma^{2} \frac{\partial \rho}{\partial x_{1}}\left(1+B_{1} B_{2} \sigma^{2} \rho-B_{2}^{2} \sigma^{2}\right) M\left(B_{1}, B_{2}\right)
$$

$\mathcal{E}\left\{h_{1}^{2} e^{i B_{1} h_{1}-i B_{2} h_{2}}\right\}$

$$
=\sigma^{2}\left(1-B_{1}^{2} \sigma^{2}+2 B_{1} B_{2} \sigma^{2} \rho-B_{2}^{2} \sigma^{2} \rho^{2}\right) M\left(B_{1}, B_{2}\right)
$$

$E\left\{h_{1} h_{2} e^{i B_{1} h_{1}-i B_{2} h_{2}}\right\}$

$$
=\sigma^{2}\left[\rho-B_{1}^{2} \sigma^{2} \rho+B_{1} B_{2} \sigma^{2}\left(1+\rho^{2}\right)-B_{2}^{2} \sigma^{2} \rho\right] M\left(B_{1}, B_{2}\right)
$$

$\mathscr{E}\left\{\gamma_{1} \gamma_{2} e^{i B_{1} h_{1}-i B_{2} h_{2}}\right\}$

$$
=\sigma^{2}\left(\frac{\partial^{2} \rho}{\partial x_{1} \partial x_{2}}+B_{1} B_{2} \sigma^{2} \frac{\partial \rho}{\partial x_{1}} \frac{\partial \rho}{\partial x_{2}}\right) M\left(B_{1}, B_{2}\right) .
$$

\section{Appendix B. Normalized Mean Square Deviation of $\left|E_{j 0}\right|$}

The expression for the normalized mean square amplitude deviation of $\left|E_{j 0}\right|$ can be written in the form

$$
\begin{array}{r}
\operatorname{msd}\left(\left|E_{j 0}\right|\right)=\left\{\frac { \sqrt { a } } { \pi } e ^ { - \sqrt { 2 } B _ { 0 } } \left[\sum_{k=1}^{5} A_{j k} A_{j k}^{*} P_{k k}+2\left(Q_{j 31}\right.\right.\right. \\
\left.\left.+Q_{j 41}+Q_{j 43}+Q_{j 52}+Q_{j 61}+Q_{j 71}+Q_{j 91}+Q_{j 111}\right)\right] \\
\left.-\left(L_{j}^{2}+M_{j}^{2}\right)\right\}\left(L_{j}^{2}+M_{j}^{2}\right)^{-1} .
\end{array}
$$

The definitions of the $A_{j k}$ are recorded in (20). The appropriate expressions for the $Q_{j m n}$ are given by

$$
Q_{j m n}=\operatorname{Real}\left\{A_{j m} A_{j n} P_{m n}\right\}
$$

where the various $P_{m n}$ are defined as follows

$P_{11}=I_{1}$

$P_{22}=\frac{\delta^{2}}{2}\left(\frac{1}{a} I_{2}-I_{3}\right)$

$P_{33}=\frac{2}{B_{0}^{2}} I_{5}-\frac{\delta^{4}}{a} I_{4}+\frac{\delta^{4}}{4} I_{6}+\frac{2 a}{B_{0}^{2}}\left(\frac{1}{a} I_{2}+I_{3}\right)+\frac{1}{B_{0}^{2}} I_{1}$

$P_{44}=\sigma^{2} I_{7}+B_{0}^{2} \sigma^{4} I_{1}+B_{0}^{2} \sigma^{4} I_{10}$

$P_{55}=2 \frac{\sigma^{2}}{\delta^{2}} I_{7}-4 \frac{\sigma^{2}}{\delta^{2}} I_{9}-4 \frac{B_{0}^{2} \sigma^{4}}{\delta^{2}} I_{11}$

$P_{31}=\frac{\delta^{2}}{2 a} I_{2}+i \frac{\delta^{2}}{2} I_{3}+\frac{1}{B_{0}} \frac{\sqrt{2}}{2}(1+i) I_{1}$

$P_{41}=-\frac{\sqrt{2}}{2} B_{0} \sigma^{2}\left[(1-i) I_{1}+(1+i) I_{7}\right]$

$P_{43}=-\sigma^{2}\left[(1-i) I_{2}-i I_{1}+(1+i) I_{8}\right.$

$\left.+I_{7}-(1+i) a I_{3}+(1-i) a I_{9}\right]$

$P_{52}=-\sqrt{2} B_{0} \sigma^{2} I_{9}$

$P_{61}=i \sqrt{2} B_{0} \sigma^{2} I_{9}$

$P_{71}=\left(\sigma^{2}-i B_{0}^{2} \sigma^{4}\right) I_{1}+2 B_{0}^{2} \sigma^{4} I_{7}+i B_{0}^{2} \sigma^{4} I_{10}$

$P_{91}=i \sigma^{2}\left[(1+i) I_{2}-a(1-i) I_{3}+i I_{1}\right.$

$$
\left.-(1-i) I_{8}-a(1+i) I_{9}-I_{7}\right]
$$

$P_{111}=\frac{2}{B_{0}^{2}} I_{5}+i \frac{3}{2} \frac{\delta^{4}}{a} I_{4}-\frac{\delta^{4}}{4} I_{6}+\frac{2}{B_{0}^{2}}(1+i) I_{2}$

$$
-\frac{2 a}{B_{0}^{2}}(1-i) I_{3}+i \frac{3}{B_{0}^{2}} I_{1} \text {. }
$$

The $I_{k}$ appearing in these last equations are defined by

$$
\begin{aligned}
& I_{1}=I(a, b ; 0,0) \\
& I_{2}=I(a, b ; 1,0) \\
& I_{3}=I(a, b ; 0,1) \\
& I_{4}=I(a, b ; 1,1) \\
& I_{5}=I(a, b ; 2,0) \\
& I_{6}=I(a, b ; 0,2) \\
& I_{7}=I(a+1, b ; 0,0) \\
& I_{8}=I(a+1, b ; 1,0) \\
& I_{9}=I(a+1, b ; 0,1) \\
& I_{10}=I(a+2, b ; 0,0) \\
& I_{11}=I(a+2, b ; 0,1)
\end{aligned}
$$


where $I(p, q ; j, k)$ is the series

$$
I(p, q ; j, k)=\pi D(j) D(k) \sum_{r=0}^{\infty} \frac{q^{r}}{r !}(p+r)^{-\left(k+\frac{1}{2}\right)}
$$

where

$$
\begin{aligned}
& D(j)=\frac{(2 j-1) !}{4^{j}(j-1) !} \\
& D(0)=\frac{1}{2} .
\end{aligned}
$$

Finally, the functions $L_{j}$ and $M_{j}$ in $(\mathrm{B}-1)$ are obtained from

$$
\begin{gathered}
L_{j}+i M_{j}=\frac{1}{2} e^{-\frac{\sqrt{2}}{2} B_{0}} \exp \left[i\left(\frac{\sqrt{2}}{2} B_{0}-\frac{1}{2} B_{0}^{2} \sigma^{2}\right)\right] \\
{\left[A_{j 1}+A_{j 3}\left(\frac{\sqrt{2}}{B_{0}}\right)(1+i)-A_{j 4}\left(\frac{\sqrt{2}}{2} B_{0} \sigma^{2}\right)(1-i)\right.} \\
\left.+A_{j 7} \sigma^{2}\left(1-i B_{0}^{2} \sigma^{2}\right)-A_{j 9}\left(2 \sigma^{2}\right)+A_{j 11}\left(i \frac{8}{B^{2}}\right)\right] .
\end{gathered}
$$

\section{References}

Baños, A., Jr., and J. P. Wesley (1953), The horizontal dipole in a conducting halfspace, Univ. of Calif., Marine Physical Laboratory, Report No. 53-33.

Baños, A., Jr., and J. P. Wesley (1954), The horizontal dipole in a conducting halfspace, Univ. of Calif., Marine Physical Laboratory, Report No. 54-33.
Davenport, W. B., Jr., and W. L. Root (1958), Random signals and noise, section 6-4 (McGraw-Hill Book Co., Inc., New York, N.Y.)

Defant, A. (1961), Physical oceanography, II, Part I (Pergamon Press, New York, N.Y.).

Hoffman, W. C. (1955), Scattering of electromagnetic waves from a random surface, Quart. Appl. Math. XIII, 291-304.

Moore, R. K., and W. E. Blair (Nov.-Dec. 1961), Dipole radiation in a conducting half space, J. Research NBS 65D (Radio Prop.), No. 6, 547-563

Norton, K. A. (Sept. 1937), The physical reality of space and surface waves in the radiation field of radio antennas, Proc. IRE 25, 1192-1202.

Sommerfeld, A. (Mar. 1909), On the propagation of electromagnetic waves over a plane earth, Ann. der Phys. 28, $665-736$.

Sommerfeld, A. (1926), On the propagation of waves in wireless telegraphy, Ann. der Phys. Ser. 4, 81, 1135-1153.

Stratton, J. A. (1941), Electromagnetic theory, sec. 8.14 (McGraw-Hill Book Co., Ine., New York, N.Y.).

Weyl, H. (Nov. 1919), Propagation of electromagnetic waves over a plane conductor, Ann. der Phys. Ser. 4, 60, 481-500.

Wait, J. R. (May 1959a), Radiation from a small loop immersed in a semi-infinite conducting medium, Can. J. Phys. 3\%, 672-674.

Wait, J. R. (June 1959b). The calculation of the field in a homogeneous conductor with a wavy interface, Proc. IRE 4\%, 1155-1156.

\section{Additional References}

Rice, S. O. (1951), Reflection of electromagnetic waves from slightly rough surfaces, Theory of electromagnetic waves (Symposium), Interscience Publication.

Senior, T. B. A. (1960a), Impedance boundary conditions for imperfectly conducting surfaces, Appl. Sci. Res. Sect. B, 8, $418-436$.

Senior, T. B. A. (1960b), Impedance boundary conditions for statistically rough surface, Appl. Sci. Res. Sect. B, 8, $437-462$.

(Paper 67D5-287) 